\title{
Total Esophageal Gastric Dissociation for the Failed Antireflux Procedure in a Child with Microgastria
}

\author{
Kengo Hattori $^{1,2}$ Bruce Bvulani ${ }^{1,2}$ Alp Numanoglu ${ }^{1,2}$ Sharon Gail Cox ${ }^{1,2}$ Alastair J. W. Millar $r^{1,2}$ \\ ${ }^{1}$ Department of Paediatric Surgery, Red Cross War Memorial \\ Children's Hospital, Cape Town, South Africa \\ ${ }^{2}$ Department of Paediatric Surgery, University of Cape Town, \\ Cape Town, Western Cape, South Africa \\ Address for correspondence Kengo Hattori, MD, LLM, Department of \\ Paediatric Surgery, Red Cross War Memorial Children's Hospital, , \\ Klipfontein Road, Rondebosch, Cape Town 7700, South Africa \\ (e-mail: ken5hattori@gmail.com).
}

Eur J Pediatr Surg Rep 2016;4:6-9.
Abstract
Keywords
- microgastria
- gastroesophageal reflux
- total esophageal gastric dissociation
- failed fundoplication

Microgastria is a very rare anomaly characterized by a very small tubular stomach that presents with severe gastroesophageal reflux disease due to the small reservoir capacity of the stomach. We present a patient with microgastria-related reflux and a failed fundoplication who was treated with total esophageal gastric dissociation (TEGD) resulting in an excellent outcome. In our experience with this good long-term result, we would suggest that TEGD be added to the armamentarium of procedures that can be used in the treatment of microgastria.

\section{New Insights and Importance for the Pediatric Surgeon}

This report highlights microgastria-related reflux and a failed fundoplication who was treated with total esophageal gastric dissociation (TEGD) resulting in an excellent outcome. We believe this is the first report revealing the long-term outcome of TEGD being used in the treatment of microgastria.

\section{Introduction}

Microgastria is a very rare anomaly characterized by a small tubular stomach and is almost always associated with other congenital anomalies. ${ }^{1}$ To date, less than 100 cases of microgastria have been described in the literature. ${ }^{2}$

Patients invariably encounter severe gastroesophageal reflux disease (GERD) due to the small reservoir capacity of the stomach and absence of esophagogastric angle of His. Presentation is with food intolerance and failure to thrive. There have been various surgical procedures described as a choice of reconstruction for microgastria, including gastric augmentation with Roux-en-Y jejunal pouch anastomosed to the stomach, the Hunt-Lawrence pouch. ${ }^{3}$ However, adequate control of GERD remains a concern in these patients. $^{2}$

received

July 31,2015

accepted after revision

December 1, 2015

published online

January 22, 2016

$10.1055 / \mathrm{s}-0035-1571176$ ISSN 2194-7619.
TEGD first described as a salvage procedure for failed fundoplications in neurologically impaired children due to the high rate of failures of repeat operations in this group of patients, ${ }^{4}$ and can offer a superior solution to eliminate GERD.

We present a patient with microgastria treated with TEGD and esophageal-jejunal anastomosis after a failed fundoplication.

\section{Case Report}

A male baby, born at term via a normal vaginal delivery presented at 3 weeks of age with dyspnea, poor feeding, and growth below the third percentile for weight for age. There was no relevant family history and antenatal investigations did not reveal any anomalies. He was noted to have a ventriculoseptal defect (VSD) on echocardiography with

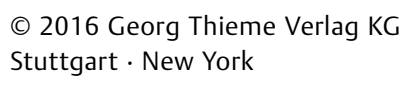

License terms

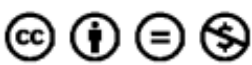


moderate left to right shunting and a respiratory tract infection for which he received treatment. He was also noted to have a left preauricular sinus, a hypoplastic left thumb and radius, and abnormal left sacral hemivertebrae but chromosomal studies were normal. He showed no neurological abnormalities and no other visible congenital anomalies.

He had repeated admissions for recurrent lower respiratory infections over the following month. Milk scan (nuclear scintigraphy with technetium labeled formula feed) and contrast study of the upper gastrointestinal tract demonstrated significant reflux with a suspected sliding hiatal hernia ( - Fig. 1). There was no improvement of the infant's general condition with various medical interventions, and at this stage, no suspicion of microgastria had been entertained.

\section{Initial Surgery}

At 3 months, he was taken to operating room for an open antireflux procedure. A long tubular stomach was observed with insufficient fundus for Nissen fundoplication and a decision was made to perform a partial anterior fundoplication. ${ }^{5}$ The spleen was thought to be mal-positioned. No hiatal hernia was observed. Postoperatively, he was commenced on feeds but continued to show clinical signs of ongoing gastroesophageal reflux. Contrast swallow revealed hold up proximal to the wrap and delayed gastric emptying. Fiberoptic esophagogastroscopy showed a reasonably snug wrap with the scope passing with ease, a tubular stomach and normal duodenum.

In view of the recurrent respiratory tract infections, one of which required intensive care ward admission and ventilation, and ongoing reflux demonstrated on radiographic contrast studies, it was thought that the fundoplication procedure had

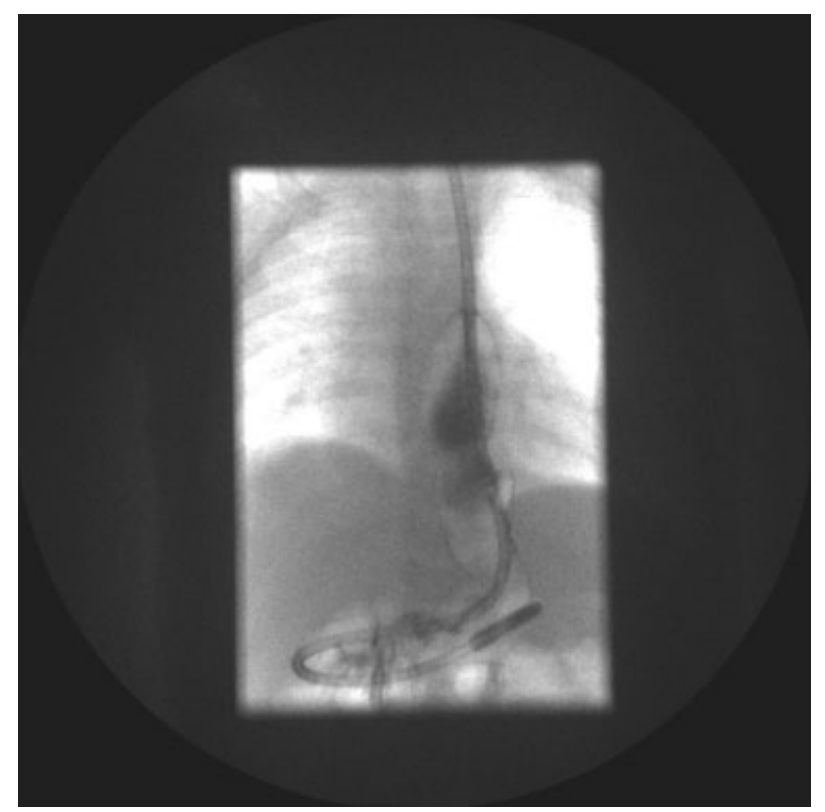

Fig. 1 Contrast swallow image at the age of 2 months demonstrating a distended distal esophagus with a small tubular stomach consistent with microgastria. Initially, the findings were thought to show significant reflux with a suspected hiatal hernia. failed. It was then decided to undertake a TEGD at 7 months of age and 4 months after the initial operation.

\section{Total Esophageal Gastric Dissociation Surgical Technique}

Midline laparotomy was performed. After taking down multiple adhesions from the previous surgery, the still intact wrap was dissected off the esophagus and the tubular stomach. The esophagus was divided at the esophagogastric junction and the stomach fundus oversewn with 5/0 PDS (Ethicon, Somerville, New Jersey, United States) suture in a single layer. The vagal nerves were not preserved. A $35-\mathrm{cm}$ Roux-en-Y segment of jejunum was passed posterior to the colon and stomach (retrocolic-retrogastric) and anastomosed primarily to the esophagus in an end-to-end fashion with 5/0 PDS suture. An enteroenterostomy was made between proximal jejunum and the descending loop of jejunum. A HeineMikulicz pyloroplasty was also done to achieve adequate gastric emptying (-Fig. 2).

\section{Result}

Recovery was uneventful. The child was kept on total parenteral nutrition for the first 2 weeks. He was started on gradually increasing nasojejunal feeds once the contrast meal showed no hold up or reflux and no retrograde entry of contrast into the proximal gastroduodenojejunal arm of

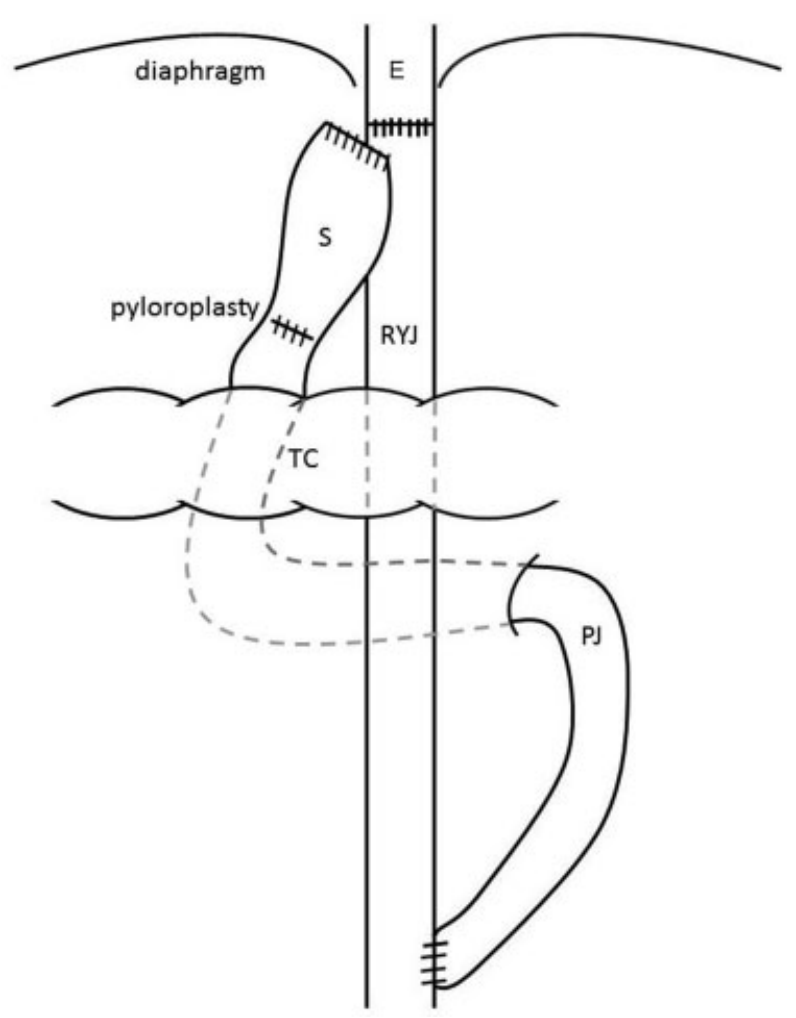

Fig. 2 Line diagram of our TEGD. E, esophagus; PJ, proximal jejunum; RYJ, Roux-en-Y segment of jejunum; S, stomach; TC, transverse colon; TEGD, total esophageal gastric dissociation. 
the Roux-en-Y loop. Oral feeds were started on day 20 postoperation and were tolerated well. He had his VSD repaired at 5 months. His respiratory symptoms had completely disappeared, and he had achieved a good recovery of all growth parameters with an age-appropriate diet tolerated without vomiting. At 4 years, 10 months of age, the latest follow-up showed his height and weight for age were both around 10th percentile $(100.5 \mathrm{~cm} / 15.5 \mathrm{~kg})$.

\section{Discussion}

Microgastria is a rare anomaly characterized by a very small tubular stomach. It has been described in patients as an isolated anomaly or in association with various degrees of limb-reduction (amelia) defects; in association with the VACTERL complex (vertebral anomalies, anorectal malformations, cardiac, trachea esophageal fistula, renal agenesis, limb anomalies); the Pierre-Robin sequence (micrognathia); and in association with other foregut developmental anomalies such as asplenia, splenic malposition, and intestinal malrotation. ${ }^{1,6-8}$

Treatment for microgastria-associated GERD has ranged from conservative expectant nonsurgical management to various forms of antireflux procedures with good long-term results being recorded from the Hunt-Lawrence jejunal pouch to augment the stomach. ${ }^{1,3,6,9-11}$ Since the first report of the Hunt-Lawrence pouch for microgastria in $1980,{ }^{11}$ several case reports have suggested such favorable outcomes compared with conservative treatments that early HuntLawrence pouch procedure is thought to be the choice of operation. ${ }^{10}$ However, in these reports, age-appropriate growth catch up was rarely achieved or otherwise nutritional support such as a feeding tube were needed. ${ }^{2,10}$ In addition, GERD remains a concern in these patients.

TEGD has been reported as achieving good outcomes not only as a salvage procedure for failed antireflux procedures in children with severe neurological impairment but as a primary antireflux procedure in those populations. ${ }^{4,12-15}$ Gatti et al showed a marked reduction in reflux symptoms and an improved quality of life with TEGD when compared with fundoplication in neurologically impaired patients. ${ }^{16}$ Buratti et al suggested TEGD be reserved only for repeated failed antireflux procedures. ${ }^{17}$ Repeat operations, however, may pose a challenge due to multiple adhesions and numerous postoperative complications may be encountered. A primary procedure would thus be less challenging, with an equally good outcome. In their follow-up of 50 patients, Lall et al showed a good outcome with a primary TEGD despite some complications. ${ }^{15}$

Upon review of the literature, we found at least two very similar cases in which the children with microgastria were treated by TEGD. ${ }^{2,15}$ In these two cases, one child was able to tolerate all food orally and a gastrostomy used for supplementary feeding could be removed, ${ }^{2}$ and the other one had been able to eat normally without any food alternation or restrictions. ${ }^{15}$ Most of the TEGD published cases have included a gastrostomy in the procedure. We believe that microgastria cases without severe neurological impairment should not need a gastrostomy in the antireflux procedures. In fact, these patients have normal swallowing abilities that enable them to feed normally after the successful antireflux procedures.

Complications that have been associated with TEGD include early anastomotic leaks, esophagojejunal anastomosis dehiscence, subphrenic collections, stricture formation, bowel obstruction from adhesions, dumping syndrome, and long-term nutritional and metabolic complications. ${ }^{15,17}$ In addition, a recent publication described esophagojejunal anastomotic stricture and gastric metaplasia as long-term complications after TEGD in the patients with esophageal atresia repair. ${ }^{18}$ In our patient, we have not encountered any complications related to the surgery.

The quality of life of patients post-TEGD has been shown to be markedly improved with reduction of reflux symptoms as with our patient. ${ }^{2,6}$ Furthermore, neurologically normal patients who underwent TEGD, even with microgastria, were reported to be tolerating full oral feeds without symptoms of dumping. ${ }^{2,15}$

We present the long-term follow-up of TEGD for microgastria. In our experience with this good result, we would suggest that TEGD be added to the armamentarium of procedures that can be used in the treatment of microgastria. Despite the high complication rates in redo operations reported in the literature, it may be the only effective antireflux surgery for recurrent microgastria-associated GERD. In addition, we suggest it can be explored as a primary treatment at the surgeon's disposal in the treatment of microgastria-associated GERD.

\section{References}

1 Ramos CT, Moss RL, Musemeche CA. Microgastria as an isolated anomaly. J Pediatr Surg 1996;31(10):1445-1447

2 Kunisaki SM, Dakhoub A, Jarboe MD, Geiger JD. Gastric dissociation for the treatment of congenital microgastria with paraesophageal hiatal hernia. J Pediatr Surg 2011;46(6): e1-e4

3 Dicken BJ, Novotny NM, Breckler FD, Yim JP, Rescorla FJ. Use of the Hunt-Lawrence pouch in congenital microgastria-a report of 2 cases. J Pediatr Surg 2010;45(11):2238-2240

4 Bianchi A. Total esophagogastric dissociation: an alternative approach. J Pediatr Surg 1997;32(9):1291-1294

5 Boix-Ochoa J. The physiologic approach to the management of gastric esophageal reflux. J Pediatr Surg 1986;21(12):1032-1039

6 Menon P, Rao KL, Cutinha HP, Thapa BR, Nagi B. Gastric augmentation in isolated congenital microgastria. J Pediatr Surg 2003; 38(10):E4-E6

7 Velasco AL, Holcomb GW III, Templeton JM Jr, Ziegler MM. Management of congenital microgastria. J Pediatr Surg 1990; 25(2):192-197

8 Laurie DE, Wakeling EL. Congenital microgastria in association with Pierre-Robin sequence. Clin Dysmorphol 2008;17(2): 143-144

9 Stewart C, Stewart M, Stewart F. Microgastria-limb reduction anomaly with total amelia. Clin Dysmorphol 2002;11(3): $187-190$

10 Jones VS, Cohen RC. An eighteen year follow-up after surgery for congenital microgastria-case report and review of literature. J Pediatr Surg 2007;42(11):1957-1960 
11 Neifeld JP, Berman WF, Lawrence W Jr, Kodroff MB, Salzberg AM. Management of congenital microgastria with a jejunal reservoir pouch. J Pediatr Surg 1980;15(6):882-885

12 Islam S, Teitelbaum DH, Buntain WL, Hirschl RB. Esophagogastric separation for failed fundoplication in neurologically impaired children. J Pediatr Surg 2004;39(3):287-291, discussion 287-291

13 Molinaro F, Bindi E, Cerchia E, Angotti R, Mariscoli F, Messina M. Esophagogastric dissociation reduces the re-operation rate for persistent gastroesophageal reflux in severely neurologically impaired children. Pediatr Surg Int 2014;30(10):997-1001

14 Morabito A, Lall A, Lo Piccolo R, et al. Total esophagogastric dissociation: 10 years' review. J Pediatr Surg 2006;41(5):919-922
15 Lall A, Morabito A, Bianchi A. "Total Gastric Dissociation (TGD)" in difficult clinical situations. Eur J Pediatr Surg 2006;16(6):396-398

16 Gatti C, di Abriola GF, Villa M, et al. Esophagogastric dissociation versus fundoplication: which is best for severely neurologically impaired children? J Pediatr Surg 2001;36(5):677-680

17 Buratti S, Kamenwa R, Dohil R, Collins D, Lavine JE. Esophagogastric disconnection following failed fundoplication for the treatment of gastroesophageal reflux disease (GERD) in children with severe neurological impairment. Pediatr Surg Int 2004;20(10):786-790

18 Gottrand M, Michaud L, Guimber D, et al. Barrett esophagus and esophagojejunal anastomotic stenosis as complications of esophagogastric disconnection in children with esophageal atresia. J Pediatr Gastroenterol Nutr 2013;57(1):93-95 